\title{
Existence and Uniqueness of Positive Solutions for Discrete Fourth-Order Lidstone Problem with a Parameter
}

\author{
Yanbin Sang, ${ }^{1,2}$ Zhongli Wei, ${ }^{2,3}$ and Wei Dong 4 \\ ${ }^{1}$ Department of Mathematics, North University of China, Taiyuan, Shanxi 030051, China \\ ${ }^{2}$ School of Mathematics, Shandong University, Jinan, Shandong 250100, China \\ ${ }^{3}$ Department of Mathematics, Shandong Jianzhu University, Jinan, Shandong 250101, China \\ ${ }^{4}$ Department of Mathematics, Hebei University of Engineering, Handan, Hebei 056021, China \\ Correspondence should be addressed to Yanbin Sang, sangyanbin@126.com
}

Received 9 January 2010; Revised 23 March 2010; Accepted 26 March 2010

Academic Editor: A. Pankov

Copyright (C) 2010 Yanbin Sang et al. This is an open access article distributed under the Creative Commons Attribution License, which permits unrestricted use, distribution, and reproduction in any medium, provided the original work is properly cited.

This work presents sufficient conditions for the existence and uniqueness of positive solutions for a discrete fourth-order beam equation under Lidstone boundary conditions with a parameter; the iterative sequences yielding approximate solutions are also given. The main tool used is monotone iterative technique.

\section{Introduction}

In this paper, we are interested in the existence, uniqueness, and iteration of positive solutions for the following nonlinear discrete fourth-order beam equation under Lidstone boundary conditions with explicit parameter $\beta$ given by

$$
\begin{gathered}
\Delta^{4} y(t-2)-\beta \Delta^{2} y(t-1)=h(t)\left[f_{1}(y(t))+f_{2}(y(t))\right], \quad t \in[a+1, b-1]_{\mathbb{Z}} \\
y(a)=0=\Delta^{2} y(a-1), \quad y(b)=0=\Delta^{2} y(b-1),
\end{gathered}
$$

where $\Delta$ is the usual forward difference operator given by $\Delta y(t)=y(t+1)-y(t), \Delta^{n} y(t)=$ $\Delta^{n-1}(\Delta y(t)),[c, d]_{\mathbb{Z}}:=\{c, c+1, \ldots, d-1, d\}$, and $\beta>0$ is a real parameter.

In recent years, the theory of nonlinear difference equations has been widely applied to many fields such as economics, neural network, ecology, and cybernetics, for details, see 
[1-7] and references therein. Especially, there was much attention focused on the existence and multiplicity of positive solutions of fourth-order problem, for example, [8-10], and in particular the discrete problem with Lidstone boundary conditions [11-17]. However, very little work has been done on the uniqueness and iteration of positive solutions of discrete fourth-order equation under Lidstone boundary conditions. We would like to mention some results of Anderson and Minhós [11] and $\mathrm{He}$ and Su [12], which motivated us to consider the BVP (1.1) and (1.2).

In [11], Anderson and Minhós studied the following nonlinear discrete fourth-order equation with explicit parameters $\beta$ and $\lambda$ given by

$$
\Delta^{4} y(t-2)-\beta \Delta^{2} y(t-1)=\lambda f(t, y(t)), \quad t \in[a+1, b-1]_{\mathbb{Z}}
$$

with Lidstone boundary conditions (1.2), where $\beta>0$ and $\lambda>0$ are real parameters. The authors obtained the following result.

Theorem 1.1 (see [11]). Assume that the following condition is satisfied

$\left(A_{1}\right) f(t, y)=g(t) w(y)$, where $g:[a+1, b-1]_{\mathbb{Z}} \rightarrow[0, \infty)$ with $\sum_{z=a+1}^{b-1} g(z)>0, w:$ $[0, \infty) \rightarrow(0, \infty)$ is continuous and nondecreasing, and there exists $\theta \in(0,1)$ such that $w(\kappa y) \geq \kappa^{\theta} w(y)$ for $\kappa \in(0,1)$ and $y \in[0, \infty)$,

then, for any $\lambda \in(0,+\infty)$, the BVP (1.3) and (1.2) has a unique positive solution $y_{\lambda}$. Furthermore, such a solution $y_{\lambda}$ satisfies the following properties:

(i) $\lim _{\lambda \rightarrow 0^{+}}\left\|y_{\lambda}\right\|=0$ and $\lim _{\lambda \rightarrow \infty}\left\|y_{\lambda}\right\|=\infty$;

(ii) $y_{\lambda}$ is nondecreasing in $\lambda$;

(iii) $y_{\lambda}$ is continuous in $\lambda$, that is, if $\lambda \rightarrow \lambda_{0}$, then $\left\|y_{\lambda}-y_{\lambda_{0}}\right\| \rightarrow 0$.

Very recently, in [12], $\mathrm{He}$ and $\mathrm{Su}$ investigated the existence, multiplicity, and nonexistence of nontrivial solutions to the following discrete nonlinear fourth-order boundary value problem

$$
\begin{gathered}
\Delta^{4} u(t-2)+\eta \Delta^{2} u(t-1)-\xi u(t)=\lambda f(t, u(t)), \quad t \in \mathbb{Z}[a+1, b+1], \\
u(a)=0=\Delta^{2} u(a-1), \quad u(b+2)=0=\Delta^{2} u(b+1),
\end{gathered}
$$

where $\Delta$ denotes the forward difference operator defined by $\Delta u(t)=u(t+1)-u(t), \Delta^{n} u(t)=$ $\Delta\left(\Delta^{n-1} u(t)\right), \mathbb{Z}[a+1, b+1]$ is the discrete interval given by $\{a+1, a+2, \ldots, b+1\}$ with $a$ and $b(a<b)$ integers, $\eta, \xi, \lambda$ are real parameters and satisfy

$$
\eta<8 \sin ^{2} \frac{\pi}{2(b-a+2)}, \quad \eta^{2}+4 \xi \geq 0, \quad \xi+4 \eta \sin ^{2} \frac{\pi}{2(b-a+2)}<16 \sin ^{4} \frac{\pi}{2(b-a+2)}, \quad \lambda>0 .
$$

For the function $f$, the authors imposed the following assumption:

$\left(B_{1}\right) f(t, x)=g(t) h(x)$, where $g: \mathbb{Z}[a+1, b+1] \rightarrow[0, \infty)$ with $\sum_{t=a+1}^{b+1} g(t)>0, h:$ $\mathbb{R} \rightarrow(0, \infty)$ is continuous and nondecreasing, and there exists $\theta \in(0,1)$ such that $h(\mu x) \geq \mu^{\theta} h(x)$ for $\mu \in(0,1)$ and $x \in[0, \infty)$. 
Their main result is the following theorem.

Theorem 1.2 (see [12]). Assume that $\left(B_{1}\right)$ holds. Then for any $\lambda \in(0,+\infty)$, the BVP (1.4) has a unique positive solution $u_{\lambda}$. Furthermore, such a solution $u_{\lambda}$ satisfies the properties (i)-(iii) stated in Theorem 1.1.

The aim of this work is to relax the assumptions $\left(A_{1}\right)$ and $\left(B_{1}\right)$ on the nonlinear term, without demanding the existence of upper and lower solutions, we present conditions for the BVP (1.1) and (1.2) to have a unique solution and then study the convergence of the iterative sequence. The ideas come from Zhai et al. [18, 19] and Liang [20].

Let $\mathbb{B}$ denote the Banach space of real-valued functions on $[a-1, b+1]_{\mathbb{Z}}$, with the supremum norm

$$
\|y\|=\sup _{t \in[a-1, b+1]_{\mathbb{Z}}}|y(t)|
$$

Throughout this paper, we need the following hypotheses:

$\left(H_{1}\right) f_{i}:[0,+\infty) \rightarrow[0,+\infty)$ are continuous and $f_{i}(y)>0$ for $y>0(i=1,2)$;

$\left(H_{2}\right) h:[a+1, b-1]_{\mathbb{Z}} \rightarrow[0,+\infty)$ with $\sum_{z=a+1}^{b-1} h(z)>0 ;$

$\left(H_{3}\right) f_{1}:[0,+\infty) \rightarrow[0,+\infty)$ is nondecreasing, $f_{2}:[0,+\infty) \rightarrow[0,+\infty)$ is nonincreasing, and there exist $\varphi(\tau), \psi(\tau)$ on interval $[a+1, b-1]_{\mathbb{Z}}$ with $\varphi:[a+1, b-1]_{\mathbb{Z}} \rightarrow(0,1)$, for all $e_{0} \in(0,1)$, there exists $\tau_{0} \in[a+1, b-1]_{\mathbb{Z}}$ such that $\varphi\left(\tau_{0}\right)=e_{0}$, and $\psi(\tau)>\varphi(\tau)$, for all $\tau \in[a+1, b-1]_{\mathbb{Z}}$ which satisfy

$$
f_{1}(\varphi(\tau) y) \geq \psi(\tau) f_{1}(y), \quad f_{2}\left(\frac{1}{\varphi(\tau)} y\right) \geq \psi(\tau) f_{2}(y), \quad \forall \tau \in[a+1, b-1]_{\mathbb{Z}}, y \geq 0
$$

\section{Two Lemmas}

To prove the main results in this paper, we will employ two lemmas. These lemmas are based on the linear discrete fourth-order equation

$$
\Delta^{4} y(t-2)-\beta \Delta^{2} y(t-1)=u(t), \quad t \in[a+1, b-1]_{\mathbb{Z}},
$$

with Lidstone boundary conditions (1.2).

Lemma 2.1 (see $[11]$ ). Let $u:[a+1, b-1]_{\mathbb{Z}} \rightarrow \mathbb{R}$ be a function. Then the nonhomogeneous discrete fourth-order Lidstone boundary value problem (2.1), (1.2) has solution

$$
y(t)=\sum_{s=a}^{b} \sum_{z=a+1}^{b-1} G_{2}(t, s) G_{1}(s, z) u(z), \quad t \in[a-1, b+1]_{\mathbb{Z}}
$$


where $G_{2}(t, s)$ given by

$$
G_{2}(t, s)=\frac{1}{\ell(1,0) \ell(b, a)}\left\{\begin{array}{ll}
\ell(t, a) \ell(b, s): & t \leq s, \\
\ell(s, a) \ell(b, t): & s \leq t,
\end{array} \quad(t, s) \in[a-1, b+1]_{\mathbb{Z}} \times[a, b]_{\mathbb{Z}}\right.
$$

with $\ell(t, s)=\mu^{t-s}-\mu^{s-t}$ for $\mu=(\beta+2+\sqrt{\beta(\beta+4)}) / 2$, is the Green's function for the second-order discrete boundary value problem

$$
\begin{gathered}
-\left(\Delta^{2} y(t-1)-\beta y(t)\right)=0, \quad t \in[a, b]_{\mathbb{Z}} \\
y(a)=0=y(b)
\end{gathered}
$$

and $G_{1}(s, z)$ given by

$$
G_{1}(s, z)=\frac{1}{b-a}\left\{\begin{array}{ll}
(s-a)(b-z): & s \leq z, \\
(z-a)(b-s): & z \leq s,
\end{array} \quad(s, z) \in[a, b]_{\mathbb{Z}} \times[a+1, b-1]_{\mathbb{Z}}\right.
$$

is the Green's function for the second-order discrete boundary value problem

$$
\begin{gathered}
-\Delta^{2} x(s-1)=0, \quad s \in[a+1, b-1]_{\mathbb{Z}} \\
x(a)=0=x(b) .
\end{gathered}
$$

Lemma 2.2 (see [11]). Let

$$
m:=\frac{\ell(1,0) \ell(b, a+1)}{(b-a) \ell^{2}(b, a)}, \quad M:=\frac{(b-a) \ell^{2}(b / 2, a / 2)}{4 \ell(1,0) \ell(b, a)} .
$$

Then, for $(t, s, z) \in[a+1, b-1]_{\mathbb{Z}}^{3}$, one has

$$
m \leq G_{2}(t, s) G_{1}(s, z) \leq M
$$

\section{Main Results}

Theorem 3.1. Assume that $\left(H_{1}\right)-\left(H_{3}\right)$ hold. Then, the BVP (1.1) and (1.2) has a unique solution $y^{*}(t)$ in $D$, where

$$
D=\left\{y \in \mathbb{B} \mid y(a)=0=y(b), y(t)>0, t \in[a+1, b-1]_{\mathbb{Z}}\right\} .
$$


Moreover, for any $x_{0}, y_{0} \in D$, constructing successively the sequences

$$
\begin{array}{r}
x_{n+1}(t)=\sum_{s=a}^{b} \sum_{z=a+1}^{b-1} G_{2}(t, s) G_{1}(s, z) h(z)\left[f_{1}\left(x_{n}(z)\right)+f_{2}\left(y_{n}(z)\right)\right], \\
t \in[a-1, b+1]_{\mathbb{Z}}, n=0,1,2, \ldots, \\
y_{n+1}(t)=\sum_{s=a}^{b} \sum_{z=a+1}^{b-1} G_{2}(t, s) G_{1}(s, z) h(z)\left[f_{1}\left(y_{n}(z)\right)+f_{2}\left(x_{n}(z)\right)\right], \\
t \in[a-1, b+1]_{\mathbb{Z}}, n=0,1,2, \ldots,
\end{array}
$$

One has $x_{n}(t), y_{n}(t)$ converge uniformly to $y^{*}(t)$ in $[a-1, b+1]_{\mathbb{Z}}$.

Proof. First, we show that the BVP (1.1) and (1.2) has a solution.

It is easy to see that the BVP (1.1) and (1.2) has a solution $y=y(t)$ if and only if $y$ is a fixed point of the operator equation

$$
A\left(y_{1}, y_{2}\right)(t)=\sum_{s=a}^{b} \sum_{z=a+1}^{b-1} G_{2}(t, s) G_{1}(s, z) h(z)\left[f_{1}\left(y_{1}(z)\right)+f_{2}\left(y_{2}(z)\right)\right], \quad t \in[a-1, b+1]_{\mathbb{Z}}
$$

In view of $\left(H_{3}\right)$ and (3.3), $A\left(y_{1}, y_{2}\right)$ is nondecreasing in $y_{1}$ and nonincreasing in $y_{2}$. Moreover, for any $\tau \in[a+1, b-1]_{\mathbb{Z}}$, we have

$$
\begin{aligned}
A\left(\varphi(\tau) y_{1}, \frac{1}{\varphi(\tau)} y_{2}\right)(t) & =\sum_{s=a+1}^{b-1} \sum_{z=a+1}^{b-1} G_{2}(t, s) G_{1}(s, z) h(z)\left[f_{1}\left(\varphi(\tau) y_{1}(z)\right)+f_{2}\left(\frac{1}{\varphi(\tau)} y_{2}(z)\right)\right] \\
& \geq \psi(\tau) \sum_{s=a+1}^{b-1} \sum_{z=a+1}^{b-1} G_{2}(t, s) G_{1}(s, z) h(z)\left[f_{1}\left(y_{1}(z)\right)+f_{2}\left(y_{2}(z)\right)\right] \\
& =\psi(\tau) A\left(y_{1}, y_{2}\right)(t)
\end{aligned}
$$

for $t \in[a, b]_{\mathbb{Z}}$ and $y_{1}, y_{2} \in D$.

Let

$$
L=(b-a-1) \sum_{z=a+1}^{b-1} h(z)
$$


condition $\left(H_{2}\right)$ implies $L>0$. Since $f_{i}(y)>0$ for $y>0(i=1,2)$, by Lemma 2.2, we have

$$
\begin{aligned}
A(L, L) & =\sum_{s=a+1}^{b-1} \sum_{z=a+1}^{b-1} G_{2}(t, s) G_{1}(s, z) h(z)\left[f_{1}(L)+f_{2}(L)\right] \\
& \geq m\left[f_{1}(L)+f_{2}(L)\right] \sum_{s=a+1}^{b-1} \sum_{z=a+1}^{b-1} h(z) \\
& =m\left[f_{1}(L)+f_{2}(L)\right] L
\end{aligned}
$$

for $m$ in (2.1) and $L$ in (3.5).

Moreover, we obtain

$$
A(L, L) \leq M\left[f_{1}(L)+f_{2}(L)\right] L
$$

for $M$ in (2.1).

Thus

$$
m\left[f_{1}(L)+f_{2}(L)\right] L \leq A(L, L) \leq M\left[f_{1}(L)+f_{2}(L)\right] L
$$

Therefore, we can choose a sufficiently small number $e_{1} \in(0,1)$ such that

$$
e_{1} L \leq A(L, L) \leq \frac{L}{e_{1}}
$$

which together with $\left(H_{3}\right)$ implies that there exists $\tau_{1} \in[a+1, b-1]_{\mathbb{Z}}$ such that $\varphi\left(\tau_{1}\right)=e_{1}$, so

$$
\varphi\left(\tau_{1}\right) L \leq A(L, L) \leq \frac{L}{\varphi\left(\tau_{1}\right)}
$$

Since $\psi\left(\tau_{1}\right) / \varphi\left(\tau_{1}\right)>1$, we can take a sufficiently large positive integer $k$ such that

$$
\left[\frac{\psi\left(\tau_{1}\right)}{\varphi\left(\tau_{1}\right)}\right]^{k} \geq \frac{1}{\varphi\left(\tau_{1}\right)} .
$$

It is clear that

$$
\left[\frac{\varphi\left(\tau_{1}\right)}{\psi\left(\tau_{1}\right)}\right]^{k} \leq \varphi\left(\tau_{1}\right) .
$$


We define

$$
\begin{aligned}
& u_{0}(t)= \begin{cases}-\left[\varphi\left(\tau_{1}\right)\right]^{k} L: & t=a-1, b+1, \\
0: & t=a, b, \\
{\left[\varphi\left(\tau_{1}\right)\right]^{k} L:} & t \in[a+1, b-1]_{\mathbb{Z}}\end{cases} \\
& v_{0}(t)= \begin{cases}-\frac{L}{\left[\varphi\left(\tau_{1}\right)\right]^{k}}: & t=a-1, b+1, \\
0: & t=a, b, \\
\frac{L}{\left[\varphi\left(\tau_{1}\right)\right]^{k}}: & t \in[a+1, b-1]_{\mathbb{Z}} .\end{cases}
\end{aligned}
$$

Evidently, for $t \in[a, b]_{\mathbb{Z}}, u_{0} \leq v_{0}$. Take any $\lambda \in\left(0,\left[\varphi\left(\tau_{1}\right)\right]^{2 k}\right]$, then $\lambda \in(0,1)$ and $u_{0} \geq \lambda v_{0}$.

By the mixed monotonicity of $A$, we have $A\left(u_{0}, v_{0}\right) \leq A\left(v_{0}, u_{0}\right)$. In addition, combining $\left(H_{3}\right)$ with (3.10) and (3.11), we get

$$
\begin{aligned}
A\left(u_{0}, v_{0}\right) & =A\left(\left[\varphi\left(\tau_{1}\right)\right]^{k} L, \frac{1}{\left[\varphi\left(\tau_{1}\right)\right]^{k}} L\right) \\
& =A\left(\varphi\left(\tau_{1}\right)\left[\varphi\left(\tau_{1}\right)\right]^{k-1} L, \frac{1}{\varphi\left(\tau_{1}\right)\left[\varphi\left(\tau_{1}\right)\right]^{k-1}} L\right) \\
& \geq \psi\left(\tau_{1}\right) A\left(\left[\varphi\left(\tau_{1}\right)\right]^{k-1} L, \frac{1}{\left[\varphi\left(\tau_{1}\right)\right]^{k-1}} L\right) \geq \cdots \\
& \geq\left[\psi\left(\tau_{1}\right)\right]^{k} A(L, L) \geq\left[\psi\left(\tau_{1}\right)\right]^{k} \varphi\left(\tau_{1}\right) L \\
& \geq\left[\varphi\left(\tau_{1}\right)\right]^{k} L=u_{0} .
\end{aligned}
$$

From $\left(H_{3}\right)$, we have

$$
\begin{aligned}
A\left(y_{1}, y_{2}\right) & =A\left(\varphi(s) \frac{y_{1}}{\varphi(s)}, \frac{1}{\varphi(s)} \varphi(s) y_{2}\right) \\
& \geq \psi(s) A\left(\frac{y_{1}}{\varphi(s)}, \varphi(s) y_{2}\right), \quad \forall s \in[a+1, b-1]_{\mathbb{Z}}, y_{1}, y_{2} \geq 0
\end{aligned}
$$

and hence

$$
A\left(\frac{y_{1}}{\varphi(s)}, \varphi(s) y_{2}\right) \leq \frac{1}{\psi(s)} A\left(y_{1}, y_{2}\right), \quad \forall s \in[a+1, b-1]_{\mathbb{Z}}, y_{1}, y_{2} \geq 0
$$


Thus, we have

$$
\begin{aligned}
A\left(v_{0}, u_{0}\right) & =A\left(\frac{L}{\left[\varphi\left(\tau_{1}\right)\right]^{k}},\left[\varphi\left(\tau_{1}\right)\right]^{k} L\right) \\
& =A\left(\frac{L}{\varphi\left(\tau_{1}\right)\left[\varphi\left(\tau_{1}\right)\right]^{k-1}}, \varphi\left(\tau_{1}\right)\left[\varphi\left(\tau_{1}\right)\right]^{k-1} L\right) \\
& \leq \frac{1}{\psi\left(\tau_{1}\right)} A\left(\frac{L}{\left[\varphi\left(\tau_{1}\right)\right]^{k-1}},\left[\varphi\left(\tau_{1}\right)\right]^{k-1} L\right) \leq \cdots \\
& \leq \frac{1}{\left[\psi\left(\tau_{1}\right)\right]^{k}} A(L, L) \leq \frac{1}{\left[\psi\left(\tau_{1}\right)\right]^{k}} \frac{L}{\varphi\left(\tau_{1}\right)} .
\end{aligned}
$$

In accordance with (3.12), we can see that

$$
A\left(v_{0}, u_{0}\right) \leq \frac{L}{\left[\varphi\left(\tau_{1}\right)\right]^{k}}=v_{0} .
$$

Construct successively the sequences

$$
u_{n}=A\left(u_{n-1}, v_{n-1}\right), \quad v_{n}=A\left(v_{n-1}, u_{n-1}\right), \quad n=1,2, \ldots
$$

By the mixed monotonicity of $A$, we have $u_{1}=A\left(u_{0}, v_{0}\right) \leq A\left(v_{0}, u_{0}\right)=v_{1}$. By induction, we obtain $u_{n} \leq v_{n}, n=1,2, \ldots$. It follows from (3.14), (3.18), and the mixed monotonicity of $A$ that

$$
u_{0} \leq u_{1} \leq \cdots \leq u_{n} \leq \cdots \leq v_{n} \leq \cdots \leq v_{1} \leq v_{0}
$$

Note that $u_{0} \geq \lambda v_{0}$, so we can get $u_{n}(t) \geq u_{0}(t) \geq \lambda v_{0}(t) \geq \lambda v_{n}(t), t \in[a, b]_{\mathbb{Z}}, n=1,2, \ldots$. Let

$$
\lambda_{n}=\sup \left\{\lambda>0 \mid u_{n}(t) \geq \lambda v_{n}(t), t \in[a, b]_{\mathbb{Z}}\right\}, \quad n=1,2, \ldots
$$

Thus, we have

$$
u_{n}(t) \geq \lambda_{n} v_{n}(t), \quad t \in[a, b]_{\mathbb{Z}}, n=1,2, \ldots,
$$

and then

$$
u_{n+1}(t) \geq u_{n}(t) \geq \lambda_{n} v_{n}(t) \geq \lambda_{n} v_{n+1}(t), \quad t \in[a, b]_{\mathbb{Z}}, n=1,2, \ldots
$$

Therefore, $\lambda_{n+1} \geq \lambda_{n}$, that is, $\left\{\lambda_{n}\right\}$ is increasing with $\left\{\lambda_{n}\right\} \subset(0,1]$. Set $\tilde{\lambda}=\lim _{n \rightarrow \infty} \lambda_{n}$. We can show that $\tilde{\mathcal{\lambda}}=1$. In fact, if $0<\tilde{\mathcal{\lambda}}<1$, by $\left(H_{3}\right)$, there exists $\tau_{2} \in[a+1, b-1]_{\mathbb{Z}}$ such that $\varphi\left(\tau_{2}\right)=\tilde{\mathcal{\lambda}}$. Consider the following two cases. 
(i) There exists an integer $N$ such that $\lambda_{N}=\tilde{\lambda}$. In this case, we have $\lambda_{n}=\tilde{\lambda}$ for all $n \geq N$ holds. Hence, for $n \geq N$, it follows from (3.4) and the mixed monotonicity of $A$ that

$$
u_{n+1}=A\left(u_{\mathrm{n}}, v_{n}\right) \geq A\left(\tilde{\jmath} v_{n}, \frac{1}{\tilde{\jmath}} u_{n}\right)=A\left(\varphi\left(\tau_{2}\right) v_{n}, \frac{1}{\varphi\left(\tau_{2}\right)} u_{n}\right) \geq \psi\left(\tau_{2}\right) A\left(v_{n}, u_{n}\right)=\psi\left(\tau_{2}\right) v_{n+1}
$$

By the definition of $\lambda_{n}$, we have

$$
\lambda_{n+1}=\tilde{\lambda} \geq \psi\left(\tau_{2}\right)>\varphi\left(\tau_{2}\right)=\tilde{\lambda}
$$

This is a contradiction.

(ii) For all integer $n, \lambda_{n}<\tilde{\lambda}$. In this case, we have $0<\lambda_{n} / \tilde{\lambda}<1$. In accordance with $\left(H_{3}\right)$, there exists $\theta_{n} \in[a+1, b-1]_{\mathbb{Z}}$ such that $\varphi\left(\theta_{n}\right)=\lambda_{n} / \tilde{\lambda}$. Hence, combining (3.4) with the mixed monotonicity of $A$, we have

$$
\begin{aligned}
u_{n+1} & =A\left(u_{n}, v_{n}\right) \geq A\left(\lambda_{n} v_{n}, \frac{1}{\lambda_{n}} u_{n}\right) \\
& =A\left(\frac{\lambda_{n}}{\tilde{\jmath}} \tilde{\jmath} v_{n}, \frac{u_{n}}{\left(\lambda_{n} / \tilde{\lambda}\right) \tilde{\jmath}}\right)=\mathrm{A}\left(\varphi\left(\theta_{n}\right) \varphi\left(\tau_{2}\right) v_{n}, \frac{u_{n}}{\varphi\left(\theta_{n}\right) \varphi\left(\tau_{2}\right)}\right) \\
& \geq \psi\left(\theta_{n}\right) A\left(\varphi\left(\tau_{2}\right) v_{n}, \frac{u_{n}}{\varphi\left(\tau_{2}\right)}\right) \geq \psi\left(\theta_{n}\right) \psi\left(\tau_{2}\right) A\left(v_{n}, u_{n}\right) \\
& =\psi\left(\theta_{n}\right) \psi\left(\tau_{2}\right) v_{n+1} .
\end{aligned}
$$

By the definition of $\lambda_{n}$, we have

$$
\lambda_{n+1} \geq \psi\left(\theta_{n}\right) \psi\left(\tau_{2}\right)>\varphi\left(\theta_{n}\right) \psi\left(\tau_{2}\right)=\frac{\lambda_{n}}{\tilde{l}} \psi\left(\tau_{2}\right) .
$$

Let $n \rightarrow \infty$, we have $\tilde{\mathcal{\lambda}} \geq(\tilde{\mathcal{\lambda}} / \tilde{\mathcal{\lambda}}) \psi\left(\tau_{2}\right)>(\tilde{\mathcal{\lambda}} / \tilde{\mathcal{\lambda}}) \varphi\left(\tau_{2}\right)=\varphi\left(\tau_{2}\right)=\tilde{\lambda}$, and this is also a contradiction. Hence, $\lim _{n \rightarrow \infty} \lambda_{n}=1$.

Thus, combining (3.20) with (3.22), we have

$$
0 \leq u_{n+l}(t)-u_{n}(t) \leq v_{n}(t)-u_{n}(t) \leq v_{n}(t)-\lambda_{n} v_{n}(t)=\left(1-\lambda_{n}\right) v_{n}(t) \leq\left(1-\lambda_{n}\right) v_{0}(t)
$$

for $t \in[a, b]_{\mathbb{Z}}$, where $l$ is a nonnegative integer. Thus,

$$
\left\|u_{n+l}-u_{n}\right\| \leq\left\|v_{n}-u_{n}\right\| \leq\left(1-\lambda_{n}\right) v_{0} .
$$

Therefore, there exists a function $y^{*} \in D$ such that

$$
\lim _{n \rightarrow \infty} u_{n}(t)=\lim _{n \rightarrow \infty} v_{n}(t)=y^{*}(t) \quad \text { for } t \in[a-1, b+1]_{\mathbb{Z}} .
$$


By the mixed monotonicity of $A$ and (3.20), we have

$$
u_{n+1}(t)=A\left(u_{n}(t), v_{n}(t)\right) \leq A\left(y^{*}(t), y^{*}(t)\right) \leq A\left(v_{n}(t), u_{n}(t)\right)=v_{n+1}(t) .
$$

Let $n \rightarrow \infty$ and we get $A\left(y^{*}(t), y^{*}(t)\right)=y^{*}(t), t \in[a-1, b+1]_{\mathbb{Z}}$. That is, $y^{*}$ is a nontrivial solution of the BVP (1.1) and (1.2).

Next, we show the uniqueness of solutions of the BVP (1.1) and (1.2). Assume, to the contrary, that there exist two nontrivial solutions $y_{1}$ and $y_{2}$ of the BVP (1.1) and (1.2) such that $A\left(y_{1}(t), y_{1}(t)\right)=y_{1}(t)$ and $A\left(y_{2}(t), y_{2}(t)\right)=y_{2}(t)$ for $t \in[a-1, b+1]_{\mathbb{Z}}$. According to (3.9), we can know that there exists $0<\eta \leq 1$ such that $\eta y_{2}(t) \leq y_{1}(t) \leq(1 / \eta) y_{2}(t)$ for $t \in[a, b]_{\mathbb{Z}}$. Let

$$
\eta_{0}=\sup \left\{0<\eta \leq 1 \mid \eta y_{2} \leq y_{1} \leq \frac{1}{\eta} y_{2}\right\}
$$

Then $0<\eta_{0} \leq 1$ and $\eta_{0} y_{2}(t) \leq y_{1}(t) \leq\left(1 / \eta_{0}\right) y_{2}(t)$ for $t \in[a, b]_{\mathbb{Z}}$.

We now show that $\eta_{0}=1$. In fact, if $0<\eta_{0}<1$, then, in view of $\left(H_{3}\right)$, there exists $\bar{\tau} \in[a+1, b-1]_{\mathbb{Z}}$ such that $\varphi(\bar{\tau})=\eta_{0}$. Furthermore, we have

$$
\begin{gathered}
y_{1}=A\left(y_{1}, y_{1}\right) \geq A\left(\eta_{0} y_{2}, \frac{1}{\eta_{0}} y_{2}\right)=A\left(\varphi(\bar{\tau}) y_{2}, \frac{1}{\varphi(\bar{\tau})} y_{2}\right) \geq \psi(\bar{\tau}) A\left(y_{2}, y_{2}\right)=\psi(\bar{\tau}) y_{2}, \\
y_{1}=A\left(y_{1}, y_{1}\right) \leq A\left(\frac{y_{2}}{\eta_{0}}, \eta_{0} y_{2}\right)=A\left(\frac{y_{2}}{\varphi(\bar{\tau})}, \varphi(\bar{\tau}) y_{2}\right) \leq \frac{1}{\psi(\bar{\tau})} A\left(y_{2}, y_{2}\right)=\frac{1}{\psi(\bar{\tau})} y_{2} .
\end{gathered}
$$

In (3.34), we used the relation formula (3.16). Since $\psi(\bar{\tau})>\varphi(\bar{\tau})=\eta_{0}$, this contradicts the definition of $\eta_{0}$. Hence $\eta_{0}=1$. Therefore, the BVP (1.1) and (1.2) has a unique solution.

Finally, we show that "moreover" part of the theorem. For any initial $x_{0}, y_{0} \in D$, in accordance with (3.9), we can choose a sufficiently small number $e_{2} \in(0,1)$ such that

$$
e_{2} L \leq x_{0} \leq \frac{1}{e_{2}} L, \quad e_{2} L \leq y_{0} \leq \frac{1}{e_{2}} L
$$

It follows from $\left(H_{3}\right)$ that there exists $\tau_{3} \in[a+1, b-1]_{\mathbb{Z}}$ such that $\varphi\left(\tau_{3}\right)=e_{2}$, and hence

$$
\varphi\left(\tau_{3}\right) L \leq x_{0} \leq \frac{L}{\varphi\left(\tau_{3}\right)}, \quad \varphi\left(\tau_{3}\right) L \leq y_{0} \leq \frac{L}{\varphi\left(\tau_{3}\right)} .
$$

Thus, we can choose a sufficiently large positive integer $k$ such that

$$
\left[\frac{\psi\left(\tau_{3}\right)}{\varphi\left(\tau_{3}\right)}\right]^{k} \geq \frac{1}{\varphi\left(\tau_{3}\right)} .
$$

Define

$$
\widehat{\mathcal{u}}_{0}=\left[\varphi\left(\tau_{3}\right)\right]^{k} L, \quad \widehat{v}_{0}=\frac{L}{\left[\varphi\left(\tau_{3}\right)\right]^{k}}
$$


Obviously, $\widehat{u}_{0}<x_{0}, y_{0}<\widehat{v}_{0}$. Let

$$
\begin{gathered}
\widehat{u}_{n}=A\left(\widehat{u}_{n-1}, \widehat{v}_{n-1}\right), \quad \widehat{v}_{n}=A\left(\widehat{v}_{n-1}, \widehat{u}_{n-1}\right), \quad n=1,2, \ldots, \\
x_{n}(t)=A\left(x_{n-1}, y_{n-1}\right)(t)=\sum_{s=a}^{b} \sum_{z=a+1}^{b-1} G_{2}(t, s) G_{1}(s, z) h(z)\left[f_{1}\left(x_{n-1}(z)\right)+f_{2}\left(y_{n-1}(z)\right)\right], \\
y_{n}(t)=A\left(y_{n-1}, x_{n-1}\right)(t)=\sum_{s=a}^{b} \sum_{z=a+1}^{b-1} G_{2}(t, s) G_{1}(s, z) h(z)\left[f_{1}\left(y_{n-1}(z)\right)+f_{2}\left(x_{n-1}(z)\right)\right]
\end{gathered}
$$

for $t \in[a-1, b+1]_{\mathbb{Z}}, n=1,2, \ldots$ By induction, we get $\widehat{u}_{n} \leq x_{n} \leq \widehat{v}_{n}, \widehat{u}_{n} \leq y_{n} \leq \widehat{v}_{n}, n=1,2, \ldots$ Similarly to the above proof, it follows that there exists $\hat{y} \in D$ such that

$$
\lim _{n \rightarrow \infty} \widehat{u}_{n}=\lim _{n \rightarrow \infty} \widehat{v}_{n}=\widehat{y}, \quad A(\widehat{y}, \widehat{y})=\widehat{y} .
$$

By the uniqueness of fixed points $A$ in $D$, we get $\widehat{y}=y^{*}$. Therefore, we have

$$
\lim _{n \rightarrow \infty} x_{n}=\lim _{n \rightarrow \infty} y_{n}=y^{*}
$$

This completes the proof of the theorem.

Remark 3.2. From the proof of Theorem 3.1, we easily know that assume $\bar{y}=A(\bar{y}, \bar{x}), \bar{x}=$ $A(\bar{x}, \bar{y})$, thus, let $y_{0}=\bar{y}, x_{0}=\bar{x}$, we have

$$
y_{n}=\bar{y}, \quad x_{n}=\bar{x}, \quad n=1,2, \ldots
$$

Therefore $\bar{y}=\bar{x}=y^{*}$.

Theorem 3.3. Assume that $\left(\mathrm{H}_{2}\right)$ holds, and the following conditions are satisfied:

$\left(C_{1}\right) f:[0,+\infty) \rightarrow[0,+\infty)$ is continuous and $f(y)>0$ for $y>0$;

$\left(C_{2}\right) f:[0,+\infty) \rightarrow[0,+\infty)$ is nondecreasing;

$$
\sum_{s=a}^{b} \sum_{z=a+1}^{b-1} G_{2}(t, s) G_{1}(s, z) h(z) f(\varphi(\tau) y(z)) \geq \psi(\tau, y) \sum_{s=a}^{b} \sum_{z=a+1}^{b-1} G_{2}(t, s) G_{1}(s, z) h(z) f(y(z)),
$$

for all $\tau \in[a+1, b-1]_{\mathbb{Z}}, y \in[0,+\infty)$, where $\varphi:[a+1, b-1]_{\mathbb{Z}} \rightarrow(0,1)$, for all $e_{0} \in(0,1)$, there exists $\tau_{0} \in[a+1, b-1]_{\mathbb{Z}}$ such that $\varphi\left(\tau_{0}\right)=e_{0}$, and $\psi:[a+1, b-1]_{\mathbb{Z}} \times[0,+\infty) \rightarrow$ $(0,+\infty)$, with $\psi(\tau, y)>\varphi(\tau)$, for all $\tau \in[a+1, b-1]_{\mathbb{Z}}, y \in[0,+\infty)$; 
$\left(C_{3}\right)$ for fixed $\tau \in[a+1, b-1]_{\mathbb{Z}}$, one has

(i) $\psi(\tau, y)$ is nonincreasing with respect to $y$, and there exists $\tau_{4} \in[a+1, b-1]_{\mathbb{Z}}$ such that

$$
m f(L) \geq \varphi\left(\tau_{4}\right), \quad \frac{\psi\left(\tau_{4}, L / \varphi\left(\tau_{4}\right)\right)}{\varphi\left(\tau_{4}\right)} \geq M f(L)
$$

or

(ii) $\psi(\tau, y)$ is nondecreasing with respect to $y$, and there exists $\tau_{5} \in[a+1, b-1]_{\mathbb{Z}}$ such that

$$
m f(L) \geq \frac{\varphi\left(\tau_{5}\right)}{\psi\left(\tau_{5}, L\right)}, \quad \frac{1}{\varphi\left(\tau_{5}\right)} \geq M f(L)
$$

where $m, M$ are defined in (2.1), $L$ is defined in (3.5). Then, the BVP

$$
\begin{gathered}
\Delta^{4} y(t-2)-\beta \Delta^{2} y(t-1)=h(t) f(y(t)), \quad t \in[a+1, b-1]_{\mathbb{Z}} \\
y(a)=0=\Delta^{2} y(a-1), \quad y(b)=0=\Delta^{2} y(b-1)
\end{gathered}
$$

has a unique solution $y^{*}$.

Proof. For convenience, we still define the operator equation $A$ by

$$
A y(t)=\sum_{s=a}^{b} \sum_{z=a+1}^{b-1} G_{2}(t, s) G_{1}(s, z) h(z) f(y(z)), \quad t \in[a-1, b+1]_{\mathbb{Z}}
$$

In the following, we consider the following two cases.

(i) For fixed $\tau \in[a+1, b-1]_{\mathbb{Z}}, \psi(\tau, y)$ is nonincreasing with respect to $y$.

According to condition $\left(C_{3}\right)$ and Lemma 2.2, we can know that there exists $\tau_{4} \in[a+$ $1, b-1]_{\mathbb{Z}}$ such that

$$
\varphi\left(\tau_{4}\right) L \leq A(L) \leq \frac{\psi\left(\tau_{4}, L / \varphi\left(\tau_{4}\right)\right)}{\varphi\left(\tau_{4}\right)} L
$$

Since $\psi\left(\tau_{4}, L\right) / \varphi\left(\tau_{4}\right)>1$, we can find a sufficiently large positive integer $k$ such that

$$
\left[\frac{\psi\left(\tau_{4}, L\right)}{\varphi\left(\tau_{4}\right)}\right]^{k} \geq \frac{1}{\varphi\left(\tau_{4}\right)}
$$


For $t \in[a+1, b-1]_{\mathbb{Z}}$, we still define

$$
\begin{gathered}
u_{0}(t)=\left[\varphi\left(\tau_{4}\right)\right]^{k} L, \quad v_{0}(t)=\frac{L}{\left[\varphi\left(\tau_{4}\right)\right]^{k}}, \\
u_{n}(t)=A u_{n-1}(t), \quad v_{n}(t)=A v_{n-1}(t), \quad n=1,2, \ldots
\end{gathered}
$$

By the proof of Theorem 3.1, it is sufficient to show that

$$
u_{0} \leq u_{1} \leq v_{1} \leq v_{0}
$$

Obviously, $u_{0} \leq v_{0}$ and $u_{1} \leq v_{1}$.

In this case, it follows from conditions $\left(C_{2}\right),\left(C_{3}\right)$, and (3.49) that

$$
\begin{aligned}
u_{1} & =A u_{0}=A\left(\left[\varphi\left(\tau_{4}\right)\right]^{k} L\right) \\
& =A\left(\varphi\left(\tau_{4}\right)\left[\varphi\left(\tau_{4}\right)\right]^{k-1} L\right) \\
& \geq \psi\left(\tau_{4},\left[\varphi\left(\tau_{4}\right)\right]^{k-1} L\right) A\left(\left[\varphi\left(\tau_{4}\right)\right]^{k-1} L\right) \\
& =\psi\left(\tau_{4},\left[\varphi\left(\tau_{4}\right)\right]^{k-1} L\right) A\left(\varphi\left(\tau_{4}\right)\left[\varphi\left(\tau_{4}\right)\right]^{k-2} L\right) \\
& \geq \psi\left(\tau_{4},\left[\varphi\left(\tau_{4}\right)\right]^{k-1} L\right) \psi\left(\tau_{4},\left[\varphi\left(\tau_{4}\right)\right]^{k-2} L\right) A\left(\left[\varphi\left(\tau_{4}\right)\right]^{k-2} L\right) \\
& \geq \cdots \\
& \geq \psi\left(\tau_{4},\left[\varphi\left(\tau_{4}\right)\right]^{k-1} L\right) \psi\left(\tau_{4},\left[\varphi\left(\tau_{4}\right)\right]^{k-2} L\right) \cdots \psi\left(\tau_{4}, L\right) A(L) \\
& \geq\left[\psi\left(\tau_{4}, L\right)\right]^{k} \varphi\left(\tau_{4}\right) L \\
& \geq\left[\varphi\left(\tau_{4}\right)\right]^{k} L=u_{0} .
\end{aligned}
$$

In accordance with (3.16), we have

$$
A\left(\frac{y}{\varphi(s)}\right) \leq \frac{1}{\psi(s, y / \varphi(s))} A y,
$$


which together with condition $\left(C_{2}\right)$ and (3.48) implies that

$$
\begin{aligned}
v_{1} & =A v_{0}=A\left(\frac{L}{\left[\varphi\left(\tau_{4}\right)\right]^{k}}\right) \\
& =A\left(\frac{L}{\varphi\left(\tau_{4}\right)\left[\varphi\left(\tau_{4}\right)\right]^{k-1}}\right) \\
& \leq \frac{1}{\psi\left(\tau_{4}, L /\left[\varphi\left(\tau_{4}\right)\right]^{k}\right)} A\left(\frac{L}{\left[\varphi\left(\tau_{4}\right)\right]^{k-1}}\right) \\
& =\frac{1}{\psi\left(\tau_{4}, L /\left[\varphi\left(\tau_{4}\right)\right]^{k}\right)} A\left(\frac{L}{\varphi\left(\tau_{4}\right)\left[\varphi\left(\tau_{4}\right)\right]^{k-2}}\right) \\
& \leq \frac{1}{\psi\left(\tau_{4}, L /\left[\varphi\left(\tau_{4}\right)\right]^{k}\right)} \frac{1}{\psi\left(\tau_{4}, L /\left[\varphi\left(\tau_{4}\right)\right]^{k-1}\right)} A\left(\frac{L}{\left[\varphi\left(\tau_{4}\right)\right]^{k-2}}\right) \\
& \leq \frac{1}{\psi\left(\tau_{4}, L /\left[\varphi\left(\tau_{4}\right)\right]^{k}\right)} \frac{1}{\psi\left(\tau_{4}, L /\left[\varphi\left(\tau_{4}\right)\right]^{k-1}\right)} \cdots \frac{1}{\psi\left(\tau_{4}, L / \varphi\left(\tau_{4}\right)\right)} A(L) \\
& \leq \frac{1}{\left[\varphi\left(\tau_{4}\right)\right]^{k-1}} \frac{1}{\psi\left(\tau_{4}, L / \varphi\left(\tau_{4}\right)\right)} A(L) \\
& \leq \frac{L}{\left[\varphi\left(\tau_{4}\right)\right]^{k}}=v_{0} .
\end{aligned}
$$

(ii) For fixed $\tau \in[a+1, b-1]_{\mathbb{Z}}, \psi(\tau, y)$ is nondecreasing with respect to $y$.

In this case, by condition $\left(C_{3}\right)$ and Lemma 2.2, we can know that there exists $\tau_{5} \in$ $[a+1, b-1]_{\mathbb{Z}}$ such that

$$
\frac{\varphi\left(\tau_{5}\right) L}{\psi\left(\tau_{5}, L\right)} \leq A(L) \leq \frac{L}{\varphi\left(\tau_{5}\right)}
$$

Since $0<\varphi\left(\tau_{5}\right) / \psi\left(\tau_{5}, L / \varphi\left(\tau_{5}\right)\right)<1$, we can take a sufficiently large positive integer $k$ such that

$$
\left[\frac{\varphi\left(\tau_{5}\right)}{\psi\left(\tau_{5}, L / \varphi\left(\tau_{5}\right)\right)}\right]^{k} \leq \varphi\left(\tau_{5}\right) .
$$


For $t \in[a+1, b-1]_{\mathbb{Z}}$, we still define

$$
\begin{gathered}
u_{0}(t)=\left[\varphi\left(\tau_{5}\right)\right]^{k} L, \quad v_{0}(t)=\frac{L}{\left[\varphi\left(\tau_{5}\right)\right]^{k}} \\
u_{n}(t)=A u_{n-1}(t), \quad v_{n}(t)=A v_{n-1}(t), \quad n=1,2, \ldots
\end{gathered}
$$

We continue to prove that

$$
u_{1} \geq u_{0}, \quad v_{1} \leq v_{0} .
$$

By (3.52), combining (3.55) with the monotonicity of $\psi$, we have

$$
\begin{aligned}
u_{1} & =A u_{0}=A\left(\left[\varphi\left(\tau_{5}\right)\right]^{k} L\right) \\
& \geq \psi\left(\tau_{5},\left[\varphi\left(\tau_{5}\right)\right]^{k-1} L\right) \psi\left(\tau_{5},\left[\varphi\left(\tau_{5}\right)\right]^{k-2} L\right) \cdots \psi\left(\tau_{5}, L\right) A(L) \\
& \geq\left[\varphi\left(\tau_{5}\right)\right]^{k-1} \psi\left(\tau_{5}, L\right) A(L) \\
& \geq\left[\varphi\left(\tau_{5}\right)\right]^{k} L=u_{0} .
\end{aligned}
$$

In accordance with (3.54), combining the monotonicity of $\psi$ and (3.55), we get

$$
\begin{aligned}
v_{1} & =A v_{0}=A\left(\frac{L}{\left[\varphi\left(\tau_{5}\right)\right]^{k}}\right) \\
& \leq \frac{1}{\psi\left(\tau_{5}, L /\left[\varphi\left(\tau_{5}\right)\right]^{k}\right)} \frac{1}{\psi\left(\tau_{5}, L /\left[\varphi\left(\tau_{5}\right)\right]^{k-1}\right)} \cdots \frac{1}{\psi\left(\tau_{5}, L / \varphi\left(\tau_{5}\right)\right)} A(L) \\
& \leq \frac{1}{\left[\psi\left(\tau_{5}, L / \varphi\left(\tau_{5}\right)\right)\right]^{k}} \frac{L}{\varphi\left(\tau_{5}\right)} .
\end{aligned}
$$

An application of (3.56) yields

$$
v_{1} \leq \frac{1}{\left[\varphi\left(\tau_{5}\right)\right]^{k}} L=v_{0} .
$$

Therefore, we obtain

$$
u_{0} \leq u_{1} \leq v_{1} \leq v_{0} .
$$

For $t=a-1, b+1$, the proof is similar and hence omitted. This completes the proof of the theorem. 
Remark 3.4. In Theorem 3.1, the more general conditions are imposed on the nonlinear term than Theorem 1.1. In particular, in Theorem 3.3, $\psi(\tau, y)$ contains the variable $y$; therefore, the more comprehensive functions can be incorporated.

\section{An Example}

Example 4.1. Consider the following discrete fourth-order Lidstone problem:

$$
\begin{gathered}
\Delta^{4} y(t-2)-\Delta^{2} y(t-1)=t\left[1+y^{1 / 4}(t)+2+\frac{1}{y^{1 / 4}(t)}\right], \quad t \in[2+1,7-1]_{\mathbb{Z}} \\
y(2)=0=\Delta^{2} y(1), \quad y(7)=0=\Delta^{2} y(6) .
\end{gathered}
$$

We claim that the BVP (4.1) and (1.2) has a unique solution $y^{*}(t)$ in $D$, where

$$
D=\left\{y \in \mathbb{B} \mid y(2)=0=y(7), y(t)>0, t \in[3,6]_{\mathbb{Z}}\right\} .
$$

Moreover, for any $x_{0}, y_{0} \in D$, constructing successively the sequences

$$
\begin{aligned}
& x_{n+1}(t)=\sum_{s=2}^{7} \sum_{z=3}^{6} G_{2}(t, s) G_{1}(s, z) z\left[1+x_{n}^{1 / 4}(z)+2+\frac{1}{y_{n}^{1 / 4}(z)}\right], \quad t \in[1,8]_{\mathbb{Z}}, n=0,1,2, \ldots, \\
& y_{n+1}(t)=\sum_{s=2}^{7} \sum_{z=3}^{6} G_{2}(t, s) G_{1}(s, z) z\left[1+y_{n}^{1 / 4}(z)+2+\frac{1}{x_{n}^{1 / 4}(z)}\right], \quad t \in[2,8]_{\mathbb{Z}}, n=0,1,2, \ldots,
\end{aligned}
$$

we have $x_{n}(t), y_{n}(t)$ converge uniformly to $y^{*}(t)$ in $[2,8]_{\mathbb{Z}}$.

In fact, we choose $f_{1}(y)=1+y^{1 / 4}, f_{2}(y)=2+1 / y^{1 / 4}, h(z)=z$, thus $f_{i}(y)>0$ for $y>0(i=1,2), \sum_{z=3}^{6} h(z)=\sum_{z=3}^{6} z=18>0$. It is easy to check that $f_{1}$ is nondecreasing on $[0,+\infty), f_{2}$ is nonincreasing on $[0,+\infty)$. In addition, we set

$$
\tau= \begin{cases}3, & \varphi(\tau) \in\left(0, \frac{1}{4}\right], \\ 4, & \varphi(\tau) \in\left(\frac{1}{4}, \frac{1}{2}\right], \\ 5, & \varphi(\tau) \in\left(\frac{1}{2}, \frac{3}{4}\right], \\ 6, & \varphi(\tau) \in\left(\frac{3}{4}, 1\right),\end{cases}
$$


$\psi(\tau)=[\varphi(\tau)]^{1 / 2}$. It is easy to see that

$$
\begin{gathered}
f_{1}(\varphi(\tau) y)=1+(\varphi(\tau) y)^{1 / 4} \geq \psi(\tau)\left(1+y^{1 / 4}\right)=\psi(\tau) f_{1}(y), \quad \forall \tau \in[3,6]_{\mathbb{Z}}, y \geq 0, \\
f_{2}\left(\frac{y}{\varphi(\tau)}\right)=2+\frac{1}{(y / \varphi(\tau))^{1 / 4}} \geq \psi(\tau)\left(2+\frac{1}{y^{1 / 4}}\right), \quad \forall \tau \in[3,6]_{\mathbb{Z}}, y \geq 0 .
\end{gathered}
$$

The conclusion then follows from Theorem 3.1.

\section{Acknowledgments}

The authors were supported financially by the National Natural Science Foundation of China (10971046), the Natural Science Foundation of Shandong Province (ZR2009AM004), and the Youth Science Foundation of Shanxi Province (2009021001-2).

\section{References}

[1] R. P. Agarwal, Difference Equations and Inequalities, vol. 155, Marcel Dekker, New York, NY, USA, 1992.

[2] R. P. Agarwal, D. O'Regan, and P. J. Y. Wong, Positive Solutions of Differential, Difference and Integral Equations, Kluwer Academic Publishers, Dordrecht, The Netherlands, 1999.

[3] R. P. Agarwal, K. Perera, and D. O'Regan, "Multiple positive solutions of singular and nonsingular discrete problems via variational methods," Nonlinear Analysis: Theory, Methods \& Applications, vol. 58, no. 1-2, pp. 69-73, 2004.

[4] V. Lakshmikantham and D. Trigiante, Theory of Difference Equations: Numerical Methods and Applications, vol. 181, Academic Press, Boston, Mass, USA, 1988.

[5] W. G. Kelley and A. C. Peterson, Difference Equations: An Introduction with Applications, Academic Press, Boston, Mass, USA, 1991.

[6] J. Yu and Z. Guo, "On boundary value problems for a discrete generalized Emden-Fowler equation," Journal of Differential Equations, vol. 231, no. 1, pp. 18-31, 2006.

[7] D. B. Wang and W. Guan, "Three positive solutions of boundary value problems for $p$-Laplacian difference equations," Computers \& Mathematics with Applications, vol. 55, no. 9, pp. 1943-1949, 2008.

[8] B. Zhang, L. Kong, Y. Sun, and X. Deng, "Existence of positive solutions for BVPs of fourth-order difference equations," Applied Mathematics and Computation, vol. 131, no. 2-3, pp. 583-591, 2002.

[9] Z. He and J. Yu, "On the existence of positive solutions of fourth-order difference equations," Applied Mathematics and Computation, vol. 161, no. 1, pp. 139-148, 2005.

[10] J. V. Manojlović, "Classification and existence of positive solutions of fourth-order nonlinear difference equations," Lithuanian Mathematical Journal, vol. 49, no. 1, pp. 71-92, 2009.

[11] D. R. Anderson and F. Minhós, "A discrete fourth-order Lidstone problem with parameters," Applied Mathematics and Computation, vol. 214, no. 2, pp. 523-533, 2009.

[12] T. He and Y. Su, "On discrete fourth-order boundary value problems with three parameters," Journal of Computational and Applied Mathematics, vol. 233, no. 10, pp. 2506-2520, 2010.

[13] R. P. Agarwal and D. O'Regan, "Lidstone continuous and discrete boundary value problems," Memoirs on Differential Equations and Mathematical Physics, vol. 19, pp. 107-125, 2000.

[14] P. J. Y. Wong and R. P. Agarwal, "Multiple solutions of difference and partial difference equations with Lidstone conditions," Mathematical and Computer Modelling, vol. 32, no. 5-6, pp. 699-725, 2000.

[15] P. J. Y. Wong and R. P. Agarwal, "Results and estimates on multiple solutions of Lidstone boundary value problems," Acta Mathematica Hungarica, vol. 86, no. 1-2, pp. 137-168, 2000.

[16] P. J. Y. Wong and R. P. Agarwal, "Characterization of eigenvalues for difference equations subject to Lidstone conditions," Japan Journal of Industrial and Applied Mathematics, vol. 19, no. 1, pp. 1-18, 2002.

[17] P. J. Y. Wong and L. Xie, "Three symmetric solutions of Lidstone boundary value problems for difference and partial difference equations," Computers \& Mathematics with Applications, vol. 45, no. 6-9, pp. 1445-1460, 2003. 
[18] C.-B. Zhai and X.-M. Cao, "Fixed point theorems for $\tau$ - $\varphi$-concave operators and applications," Computers and Mathematics with Applications, vol. 59, no. 1, pp. 532-538, 2010.

[19] C. B. Zhai, W. X. Wang, and L. L. Zhang, "Generalizations for a class of concave and convex operators," Acta Mathematica Sinica, vol. 51, no. 3, pp. 529-540, 2008 (Chinese).

[20] Z. D. Liang, "Existence and uniqueness of fixed points for mixed monotone operators," Journal of Dezhou University, vol. 24, no. 4, pp. 1-6, 2008 (Chinese). 